\section{Propionibacterium acnes linked with prostatic inflammation and carcinogenesis}

A research group has cultured Propionibacterium acnes from the prostates of men undergoing radical prostatectomy for carcinoma. $P$. acnes is a slow-growing organism that is rapidly overtaken by common microbes in standard laboratory culture; these features might explain why it has not previously been found in prostatic tissue.

Cancerous prostates often have multiple foci of acute and chronic inflammation, scattered throughout the organ rather than confined to tumor boundaries. The etiology of such inflammation is unknown, although its histologic resemblance to inflammation resulting from Helicobacter pylori infection in the stomach, a precursor of gastric carcinogenesis, has led to speculation that infectious agents might have a similar role in prostate carcinogenesis. Cohen et al. suggest that indolent but persistent infection with $P$. acnes could be a contributory factor.

Intact prostate glands were removed from 34 consecutive patients with localized cancer and processed within $30 \mathrm{~min}$, taking care to avoid sites potentially exposed to surgical or skin contamination. Bacteria were found in 19 cases; $P$. acnes was predominant, occurring in 12 samples, and was the only organism to be associated with greater severity of inflammation. DNA typing and phenotypic analysis showed that all but two prostatic isolates of $P$. acnes differed from cutaneous isolates of this organism, suggesting that specific subtypes might be linked with inflammation.

Limitations of this study include the lack of control samples from healthy patients, and the observation that other, as yet unidentified, bacteria might be present. Further work should address these problems, and might lead to appropriate treatment.

Caroline Barranco

Original article Cohen RJ et al. (2005) Propionibacterium acnes associated with inflammation in radical prostatectomy specimens: a possible link to cancer evolution? J Urol 173: 1969-1974

\section{Accuracy of prostate-specific antigen testing for prostate cancer}

An important study has shown that, contrary to popular belief, there is no such thing as a 'normal' or 'abnormal' prostate-specific antigen (PSA) level. PSA screening in men aged over 50 has been widespread in the US for almost 20 years, yet no studies have ever shown it to reduce prostate cancer mortality.

Thompson and colleagues set out to measure the diagnostic accuracy of PSA for prostate cancer detection by estimating the 'receiver operating characteristic' curve. This is a plot of 1 - specificity versus sensitivity for each cut-off value of PSA. The team analyzed data from 8,575 men in the placebo group of the Prostate Cancer Prevention Trial, a prospective, multicenter, randomized, controlled trial that ran from 1993 to 2003. The value of this study population was the end-of-study prostate biopsy performed on all cancer-free men, irrespective of PSA level. This avoided verification bias and made it possible to evaluate the operating characteristics of PSA screening.

The researchers found that no cut-off value simultaneously yields both high sensitivity and high specificity, making it difficult for clinicians to know when to recommend a prostate biopsy. The commonly used cut-off value of $4.1 \mathrm{ng} / \mathrm{ml}$ detected only $20.5 \%$ of prostate cancers. However, lowering the cut-off point so that $83.4 \%$ of cases were detected would result in a false-positive rate of $61.1 \%$.

Clinicians and patients must now learn to think of PSA levels in terms of a continuum of prostate cancer risk, rather than definitive cut-off points.

Tamsin Osborne

Original article Thompson IM et al. (2005) Operating characteristics of prostate-specific antigen in men with an initial PSA level of $3.0 \mathrm{ng} / \mathrm{ml}$ or lower. JAMA 294: 66-70

\section{New treatment strategy for metastatic renal cell carcinoma}

Aass and colleagues in Europe have published the results of a randomized phase II/III trial comparing combination therapy with 13-cis-retinoic acid and interferon- $\alpha 2 \mathrm{a}$, to interferon- $\alpha 2 \mathrm{a}$ alone, in patients with progressive metastatic renal cell carcinoma. This disease is known to be highly resistant to therapy and patients typically have poor prognosis. There is therefore an urgent need for effective new treatment strategies.

A total of 320 patients with progressive metastatic renal cell carcinoma were 